\title{
PLASMACHEMICAL SYNTHESIS OF NANODISPERSED SILICON POWDER AND ITS USE AS ANODE IN LITHIUM-ION BATTERY
}

\author{
A.A.Popovich ${ }^{1}$, Wang Qingsheng ${ }^{1,2}$, P.A.Novikov ${ }^{1,2}$, N.E.Ozerskoi ${ }^{1,2} *$ \\ ${ }^{1}$ Peter the Great St. Petersburg Polytechnic University, 195251, Russia, Saint-Petersburg, yл. \\ Polytechnicheskaya street, 29 \\ ${ }^{2}$ Zhejang Changxing CHN/RUS New Energy and Material Technology Reseach Institute, 313100, \\ China, Zhejiang, Changxing County, Mingzhu Road Block B ENV Mansion №1278
}

Keywords: Nanodispersed silicon powder, lithium-ion battery, plasmachemical synthesis.

Abstract: In the work we investigated in detail the process of obtaining nanosized spherical silica powder from micron powder fragmental forms using inductively coupled argon plasma. In the course of research, the phase composition, the morphology of the particles, particle size distribution and specific surface area of the powder was studied. The resulting silicon powder was used as the anode material for the lithium-ion battery. The assembled model of the battery was investigated using the process of galvanostatic charge/discharge, cyclic voltammetry. The working electrode showed a capacity of $2056 \mathrm{mAh} / \mathrm{g}$ during charge and $1977 \mathrm{mAh} / \mathrm{g}$ during discharge, respectively.

\section{Introduction}

Currently, environmentally friendly modes of transport are being strengthened. Internal combustion engines that use hydrocarbon raw materials as fuel are replaced by environmentally friendly reusable energy sources based on the reversibility of internal chemical processes.

Lithium-ion batteries (LIBs) at this stage of development of science and technology are unambiguous leaders among the chemical current sources used in portable electronics, hybrid and electric transport in their functional characteristics. Currently, the anode material of LIBs, in most cases, is graphite, which has a theoretical specific capacity of $372 \mathrm{mAh} / \mathrm{g}$ and high cyclic stability. The desire to improve the performance of LIBs leads to the study of alternative materials and additives as anodes: nanoscale allotropic modifications of carbon [1-3], a combination of carbonaceous materials with different materials having an increased specific capacity, such as silicon, tin, germanium, manganese, zinc and their oxides [5-11]. The most promising anode material is considered to be silicon, due to the high theoretical specific capacity- $4200 \mathrm{mAh} / \mathrm{g}$, for compound Li4.4Si (Li22Si5). Schematically, the process of formation of lithium-silicon system intermetallic compounds can be represented as follows:

$$
x \mathrm{Li}^{+}+x e^{-}+\mathrm{Si} \rightarrow \mathrm{Li}_{x} \mathrm{Si}
$$

Based on the phase diagram of the lithium-silicon system [4], the following stable compounds can be distinguished, which are formed in the process of silicon anode casting: Li12Si7, Li7Si3, 


\section{Li3.25Si (Li13Si4), Li3.75Si (Li15Si4), Li4.4Si (Li22Si5).}

A significant disadvantage that prevents the industrial introduction of silicon is a 4-fold increase in its volume during the formation of intermetallic compounds by reaction (1), which leads to the destruction of silicon particles and the loss of electrical contact (Fig.1.) [4].

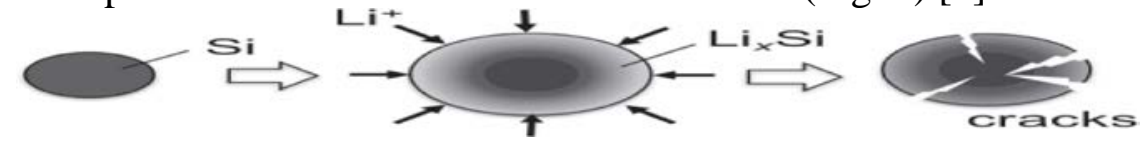

Figure 1- Schematic representation of the process of casting silicon particles larger than $150 \mathrm{~nm}$ in the process of charge/discharge [5].

Due lithation process solid-electrolyte interphase (SEI) is violated, which leads to degradation of the battery. Experimental and theoretical studies have shown that nanoscale silicon particles smaller than $150 \mathrm{~nm}$ can be effective in solving the problem of mechanical stress and avoid particle failure. [6]

To obtain nanoscale particles, several different methods are described in the literature: chemical vapor deposition (CVD) [12, 13], laser ablation [14], magnesia-thermal reduction [15], and powder spraying in a low-temperature inductively coupled plasma flow [16].

This paper presents the results of obtaining nanoscale silicon by spraying in a flame of inductively coupled plasma, as well as its use as an anode material of a lithium-ion battery.

\section{Experiment}

\subsection{Synthesis of nanosized silicon powder}

Inductively coupled plasma is a gas discharge formed inside the discharge chamber, excited by a high-frequency alternating magnetic field using an induction coil. The high plasma temperature ( 103-104K) ensures the transition of all initial substances to the gaseous ionized state, which, accordingly, leads to a high rate of interaction and rapid reaction. In this paper, the synthesis was carried out using a TEKNA Tek-15 inductively coupled plasma unit with an installed reactor to produce nanosized powders. A schematic representation of the installation is shown in figure 2 .

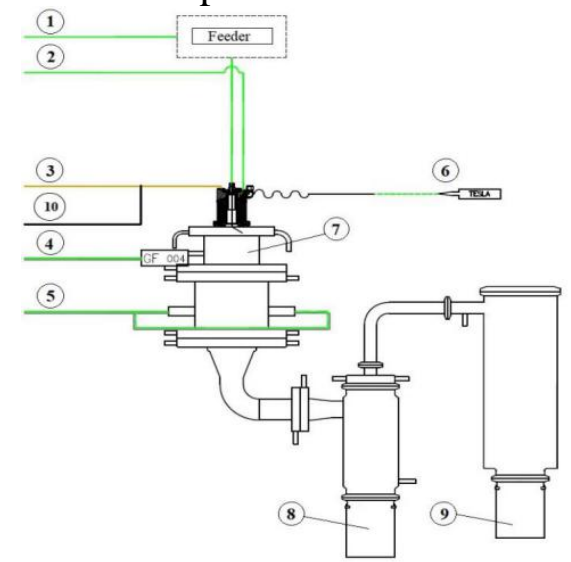

Figure 2 - Schematic representation of the reactor setup system TEKNA Tek-15. 1- Carrier gas; 2Central gas; 3- Sheath gas; 4- Quench gas\#1; 5- Quench gas\#2; 6- Starter; 7- Nanoreactor TekNano; 8- Cyclone canister; 9- Filter canister; 10- Option gas;

Silicon was used as the initial powder (Xuzhou LingYun Silicon Industry Co. Ltd, 99.99\%) and having a particle size of $\mathrm{D} 50=12 \mu \mathrm{m}$. In the gas system of the plant such gases as $\mathrm{Ar}$ (99.999\%) and H2 (99.999\%) were used. Argon was used as a carrier, central, sheath and quench gases. Hydrogen was used as an option gas to maintain the combustion of argon plasma during operation of the plant at high power. 
The initial silicon powder was dried in a vacuum furnace at $80^{\circ} \mathrm{C}$ for 12 hours before synthesis in the plant. The powder loading was $33 \mathrm{~g}$. After loading, the gas system of the plant was purged by argon to remove air from the system. Then was established the feed rate of the powder in the plasma torch, which amounted to $495 \mathrm{~g} /$ hour, by adjusting the stroke and frequency of vibration of the powder feeder. Before ignition of the plasma has been installed the cost of gases: sheath - 35 slpm, central - 10 slpm and quench \#1 - 30 slpm. Then, when the unit capacity has reached $1.4 \mathrm{~kW}$, ignition of the plasma was produced. When the power reached $10 \mathrm{~kW}$, the gas system began to supply hydrogen while increasing the power to the maximum possible. At maximum power (15 kW) hydrogen consumption was 4 slpm. After that, the quench gas\#2 was fed into the system with a flow rate of $50 \mathrm{slpm}$. The pressure in the reactor of the plant was set at the level of 1 atmosphere. Then powder flow was activated with the setting of a carrier gas with a flow rate of 7 slpm into a zone of low-temperature plasma. The synthesis time was $4 \mathrm{~min}$. After it was done purging the system with argon to remove residual hydrogen. Were then removed containers of the cyclone and filter. From the first $10.3 \mathrm{~g}$ of powder was extracted, from the second $10.6 \mathrm{~g}$.

\subsection{Material characterization}

The morphology of the powder was studied using a scanning electron microscope (Tescan Maia 3) with a fluorescent radiation detector (Bruker X-Flash 6/10). X-ray diffractometer Bruker D8 Advance was used for $\mathrm{x}$-ray analysis using $\mathrm{Cu} \mathrm{K \alpha}(1,5406 \AA)$ radiation in the $2 \theta$ range of $10^{\circ}-80^{\circ}$. Using a laser diffractometer (Malvern Mastersizer 3000) the granulometric composition of the obtained powder was investigated. Also, the analysis of the surface area of the resulting powder was carried out on the unit Micrometrics ASAP 2460.

\subsection{Electrochemical measurements}

The resulting silicon powder was mixed with a conductive additive (carbon) and a binder (polyvinylidene fluoride, PVDF) in a solvent (N-methyl-2-pyrrolidone, NMP ) in an experimentally selected ratio (67.5:13.2:19.3). The resulting slurry was deposited on a copper foil $(25 \mu \mathrm{m})$ using a coating testing machine (ShangDong Zhong Yi Equipment Co.,Ltd). The working electrodes were assembled into coin-cells CR2032 in a high purity Ar-filled glovebox (Vigor V-lab, O2 and H2O $<1$ ppm). A solution of $1.1 \mathrm{M}$ LiPF6 in ethylene carbonate, ethylmethyl carbonate and dimethyl carbonate (in a volume ratio of 1:1:1) was used as an electrolyte. Celgard $2400(25 \mu \mathrm{m})$ was used as the separator. Lithium metal was employed as the counter/reference electrode. The capacity of the obtained electrodes in the voltage range of $0.01-1.5 \mathrm{~V}$ relative to $\mathrm{Li} / \mathrm{Li}+$ was investigated by the galvanostatic charge/discharge method. The current density was set to $1 \mathrm{~A} / \mathrm{g}$ without initial stabilization cycles (BTS NEWARE CT-3008). The assembled model was also studied using cyclic voltammetry in the voltage range $0.01-1.5 \mathrm{~V}$ at a scanning rate of $0.1 \mathrm{mV} / \mathrm{sec}$ (AMETEK 1400).

\section{Results and discussion}

\subsection{Characteristics of the obtained silicon powder.}

Plasma is a high-temperature heat-carrier, silicon powder, getting into it, first melts and then evaporates. Vapor getting in the zone, serving the quenching gases, condense first, and then crystallize.

Granulometric analysis of the resulting synthesis of silicon powder showed that D90-less than $600 \mathrm{~nm}$, D50 less than $200 \mathrm{~nm}$ and D10 less than $100 \mathrm{~nm}$. However, due to the wide particle size distribution, i.e. low selectivity of this synthesis method, particles up to $1 \mu \mathrm{m}$ in size are present in 
the powder. The particle size distribution by sample volume is shown in figure 4 .

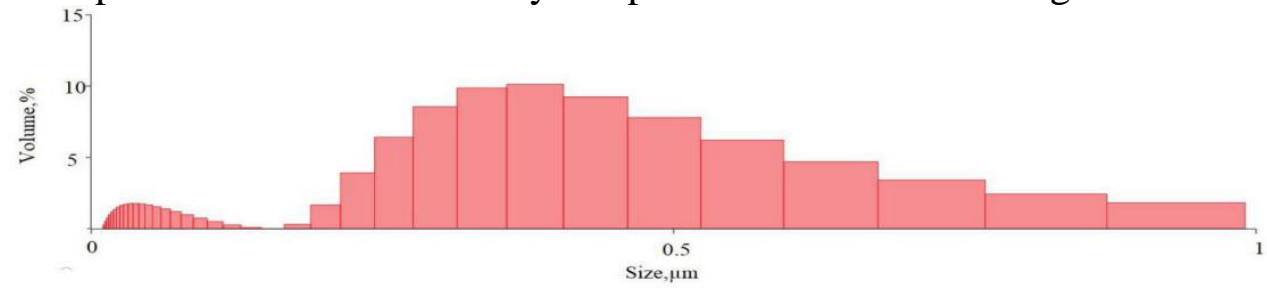

Figure 4-particle size distribution of the resulting powder by sample volume.

As a result of x-ray diffraction of the sample XRD pattern was obtained which presented in figure 5. The resulting silicon powder has a type diamond cubic lattice (Fd3m). The lattice parameter a was 5,4337 $\AA$, and the unit cell volume was 160,4359. The lattice parameters of the sample, obtained as a result of spheroidization not differ from the original powder.

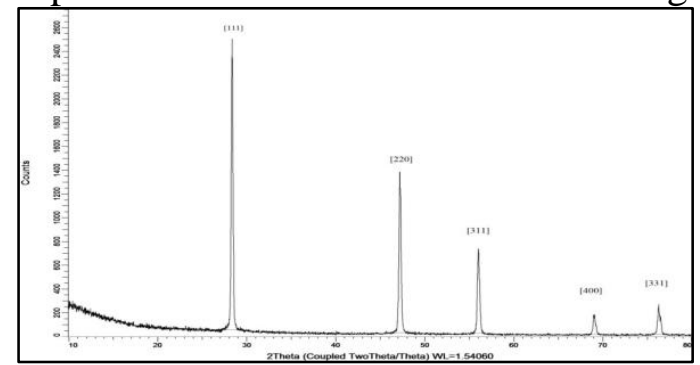

Figure 5-XRD pattern of the obtained sample of silicon powder.

The study of powder morphology on a scanning electron microscope showed that during the synthesis all the particles acquired a spherical shape (Fig. 6 (b)). Due to the low electrical conductivity of silicon, a gold coating was applied to the resulting sample prior to testing. The resulting images are shown in figure 6.

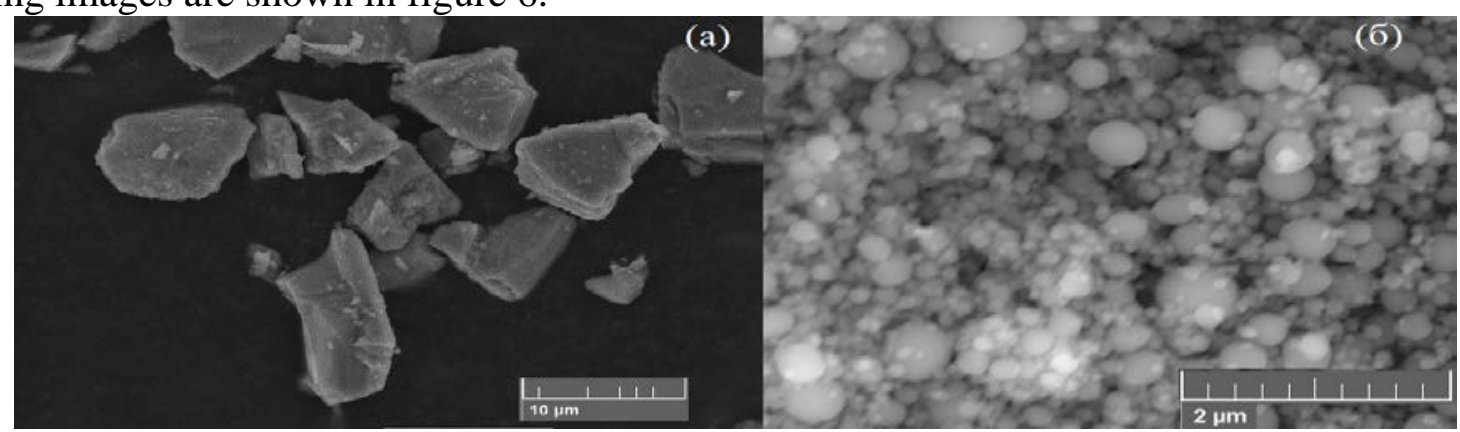

Figure 6 - Morphology of silicon powder, obtained using scanning electron microscope: a- before spheroidization; b - after spheroidization.

The analysis of micrographs obtained by scanning electron microscope showed that the average particle size was about 200-300 $\mathrm{nm}$. The results obtained are in good agreement with the data obtained using a laser diffractometer. The particles have a spherical shape, a smooth surface, but on the surface of some large particles there are smaller ones that were formed as a result of rapid cooling in the reactor.

As a result of the study of the sample on the surface area analyzer by the Brunauer-EmmettTeller (BET) method, the value of $38.70 \mathrm{~m} 2 / \mathrm{g}$ was obtained. The surface area of the initial silicon powder was $2.6 \mathrm{~m} 2 / \mathrm{g}$.

\subsection{Electrochemical characteristics of the electrode, based on the resulting silicon powder}

Profiles of galvanostatic charge/discharge were investigated in the voltage range 0.01-1.5 V with a current density of $1 \mathrm{~A} / \mathrm{g}$ (Fig.7). 


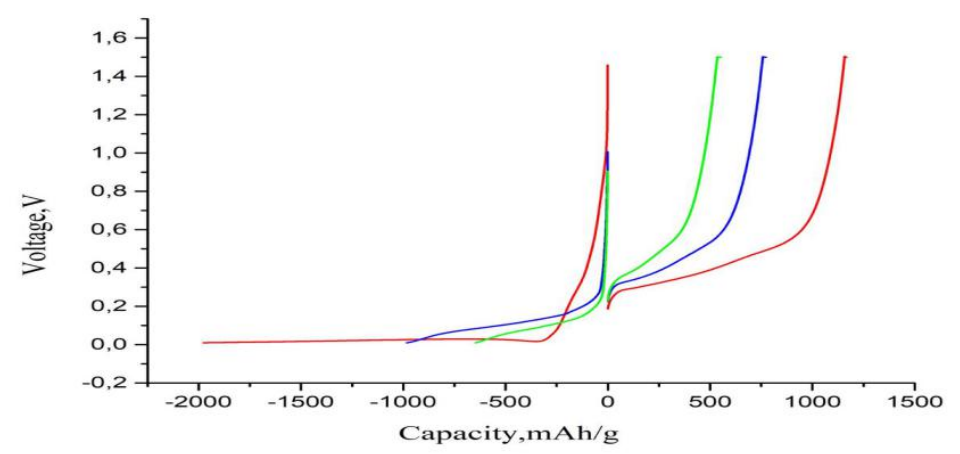

Figure 7 - Profiles of galvanostatic charge/discharge were investigated in the voltage range 0.01-1.5

$\mathrm{V}$ with a current density of $1 \mathrm{~A} / \mathrm{g}$.

The first charging and discharge capacities were $2056 \mathrm{mAh} / \mathrm{g}$ and $1977 \mathrm{mAh} / \mathrm{g}$, respectively (Fig. 9). The loss of capacity in the first cycle may be due to the formation SEI and the formation of an amorphous LixSi. During galvanostatic cycling the electrode demonstrated a casting plateau in the range $0-0.15 \mathrm{~V}$, and the dividing plateau was in the range of $0.25-0.5 \mathrm{~V}$ relative to $\mathrm{Li} / \mathrm{Li}+$. The test sample capacity decreased rapidly and after 15 cycles was $75 \mathrm{mAh} / \mathrm{g}$ and $67 \mathrm{mAh} / \mathrm{g}$ during charge and discharge, respectively. Coulomb efficiency also decreased with each subsequent cycle (Fig. 9). This is due to the fact that in the powder, which was used as an active material, there were too many particles, the size of which was more than $150 \mathrm{~nm}$, which caused an increase in the volume of silicon particles and, accordingly, the cause of degradation of the test layout.

To study the process of lithium intercalation/deintercalation in silicon anode, the cyclic voltammetry (CVA) method with a scan rate of $0.1 \mathrm{mV} / \mathrm{s}$ in the potential range from 0.01 To $1.5 \mathrm{~V}$ against $\mathrm{Li} / \mathrm{Li}+$ was used (Fig. 8).

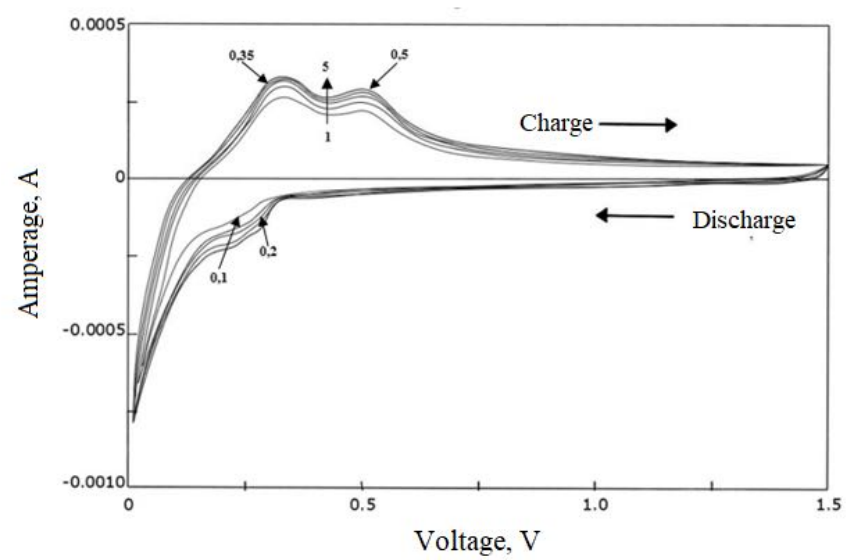

Figure 8- Profiles of cyclic voltammetry during intercalation/deintercalation of lithium into silicon anode.

The presence of a broadened cathode peak in the region of $0.1 \mathrm{~V}$ at the first cycle corresponds to the interaction of silicon with lithium and the formation of the crystalline phase Li15Si4 [17]. On the second cycle, another cathode peak of $0.2 \mathrm{~V}$ is released, indicating that the process of formation of a stable crystalline phase is preceded by the appearance of amorphous Li-Si alloys of different composition, with the general formula LixSi [7, 18]. Two anode peaks of $0.35 \mathrm{~V}$ and $0.5 \mathrm{~V}$ are observed at all CVA cycles corresponding to the Li15Si4 dividing process with the formation of amorphous silicon. All observed redox peaks are described by the following conversion reactions: 


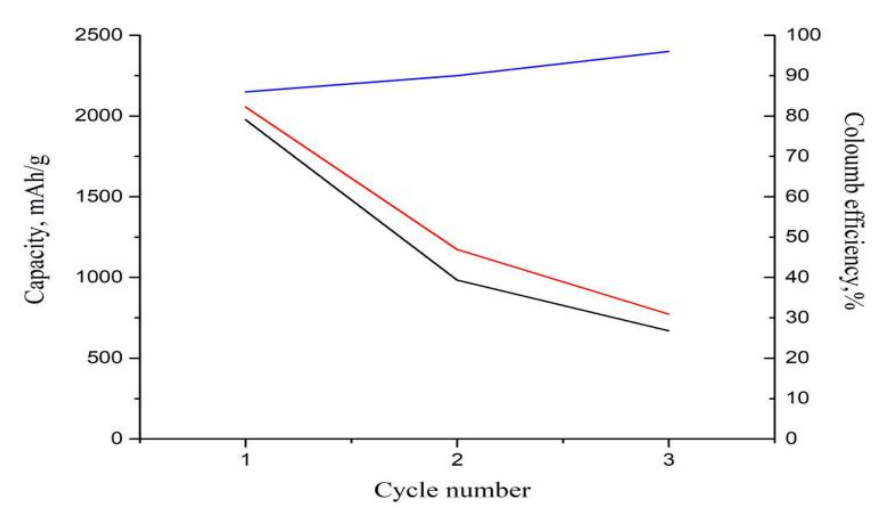

Figure 9 - Dependence of the discharge (red) and charge (blue) capacitance of the electrode, as well as its Coulomb efficiency (green) relative to the cycle.

\subsection{The study of the electrode after cycling}

After the experiment, the model containing a silicon electrode was disassembled to obtain images of the anode material degraded during cyclic tests. Figure 10 shows the images obtained by scanning electron microscope before (10 (a)) and after cycling (10(b)).

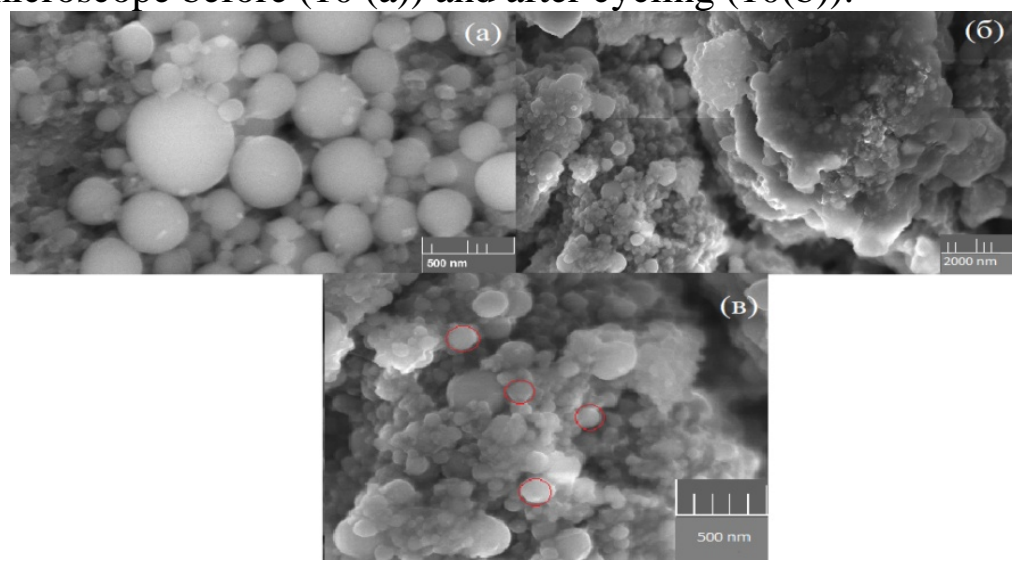

Figure 10- Images obtained by scanning electron microscope before (a) and after (b) cycling, (c) after cycling with high magnification.

From the obtained micrographs it can be seen that after cycling, in general, the working anode material has changed. As can be seen in figure 10(b) after the intercalation of lithium in the silicon structure degradation of the working electrode occurred. Before the cyclic tests, the working electrode consisted of spherical particles with an average size of $200 \mathrm{~nm}$. After cyclic tests, particles larger than $150 \mathrm{~nm}$ were destroyed and those smaller than $150 \mathrm{~nm}$ remained spherical (Fig.10 (b)). This pattern took place in the entire mass of the electrode investigated after cycling.

\section{Conclusion}

In this paper, we proposed a method of obtaining nanosized silicon for use as the active anode material of a lithium ion battery. In general, the method of obtaining silicon nanopowder in the flame of inductively coupled plasma was effective. But because of the wide distribution of particles (high selectivity) method to use the resulting silicon powder was ineffective. Since the size of the powder goes beyond $150 \mathrm{~nm}$, the working electrode showed a capacity of $2056 \mathrm{mAh} / \mathrm{g}$ during charge and $1977 \mathrm{mAh} / \mathrm{g}$ during discharge. Degradation of the studied model occurred during the 
first 10-15 cycles. To solve this problem, it is planned to improve the method of obtaining nanoscale powder in the future, as well as the possibility of coating it with various carbon structures in order to avoid an increase in the volume of particles of the powder used.

\section{References}

[1] Jiajun Dong, Tong Zhang, Dong Zhang, et al.// Nanotechnology 28 (2017).

[2] Tao Li, Cheng Wei,Yi-Min Wu, Fu-Dong Han, et al. // ACS Appl. Mater. Interfaces 7 (2015) 5107-5115.

[3] Zhuang Sun, Xuefeng Song, Peng Zhang et al. // RSC Adv. 5 (2015) 3657-3664.

[4] V.P. Nikolaev, A.G. Morachevskii, A.I. Demidov, and E.V. Bairachnyi, Zh. Prikl. Khim., 53(9), 2088-2090 (1980) in Russian

[5] Popovich, A.A., Maximov, M.Y., Novikov, P.A., Silin, A.O., Nazarov, D.V., Rumyantsev,A.M. // Russian Journal of Applied Chemistry 89, Issue 4, 1 (2016), 679-681

[6] Minoru Inaba, Masakazu Haruta, Morihiro Saito, Takayuki Doi // Electrochemistry 85 (2017) 623-629.

[7] Byeongyong Lee, Tianyuan Liu, Sun Kyung Kim et al. // Carbon 119 (2017) 438-445.

[8] I.A. Stenina, T.L. Kulova, A.M. Skundin,B. Yaroslavtsev // Rev.Adv. Mater. Sci.. 49 (2017) 140-149.

[9] Leyi Zhao, D.J. Dvorak, M.N. Obrovac // Journal of Power Sources 332 (2016) 290-298.

[10] Rui Xu, Songping Wu, Yao Du et al. // Chemical Engineering Journal. 296 (2016) 349-355.

[11] Fangcai Zheng,Guoliang Xia, Yang, et al. // Nanoscale. (2015).

[12] Sungun Wi, Hyungsub Woo, Sangheon Lee et al. // Nanoscale Research Letters. (2015).

[13] M. Ko, S. Chae, S. Jeong, P. Oh, J. Cho, ACS Nano 8 (2014) 8591-8599.

[14] A. Magasinski, P. Dixon, B. Hertzberg, A. Kvit, J. Ayala, G. Yushin, Nat. Mater 9 (2010) 353-358.

[15] U. Kasavajjula, C.S. Wang, A.J. Appleby, J. Power Sources 163 (2007) 1003-1039.

[16] Jiayin, G., Xiaobao, F., Dolbec, R., Siwen, X., Jurewicz, J., \& Boulos, M. (2010). Development of Nanopowder Synthesis Using Induction Plasma. Plasma Science and Technology, 12(2), 188-199

[17] H. Tang, J. Zhang, Y.J. Zhang, Q.Q. Xiong, Y.Y. Tong, Y. Li, et al., J. Power Sources 286 (2015) $431-437$

[18] Z.Y. Lu, J.X. Zhu, D.H. Sim, W.H. Shi, Y.Y. Tay, J. Ma, H.H. Hng, Q.Y. Yan, Electrochim. Acta 74 (2012) 176-181 